\title{
Development and operation of the microshutter array system
}

\author{
M. D. Jhabvala ${ }^{1}$, D. Franz ${ }^{1}$, T. King ${ }^{1}$, G. Kletetschka ${ }^{2}$, A. S. Kutyrev ${ }^{3}$, M. J. Li ${ }^{1}$, \\ S. E. Meyer ${ }^{1}$, S. H. Moseley ${ }^{1}$, S. Schwinger ${ }^{1}$, R. Silverberg ${ }^{1}$ \\ ${ }^{1}$ NASA Goddard Space Flight Center, Greenbelt, MD 20771, USA \\ ${ }^{2}$ Catholic University, Washington D.C. 20064, USA \\ ${ }^{3}$ University of Maryland/Cresst, College Park, MD 20742, USA
}

\begin{abstract}
The microshutter array (MSA) is a key component in the James Webb Space Telescope Near Infrared Spectrometer (NIRSpec) instrument. The James Webb Space Telescope is the next generation of a space-borne astronomy platform that is scheduled to be launched in 2013. However, in order to effectively operate the array and meet the severe operational requirements associated with a space flight mission has placed enormous constraints on the microshutter array subsystem. This paper will present an overview and description of the entire microshutter subsystem including the microshutter array, the hybridized array assembly, the integrated CMOS electronics, mechanical mounting module and the test methodology and performance of the fully assembled microshutter subsystem. The NIRSpec is a European Space Agency (ESA) instrument requiring four fully assembled microshutter arrays, or quads, which are independently addressed to allow for the imaging of selected celestial objects onto the two 4 mega pixel IR detectors. Each microshutter array must have no more than $\sim 8$ shutters which are failed in the open mode (depending on how many are failed closed) out of the $62,415(365 \times 171)$ total number of shutters per array. The driving science requirement is to be able to select up to 100 objects at a time to be spectrally imaged at the focal plane. The spectrum is dispersed in the direction of the 171 shutters so if there is an unwanted open shutter in that row the light from an object passing through that failed open shutter will corrupt the spectrum from the intended object.
\end{abstract}

Keywords: Microshutters, microshutter arrays, micromirrors, MEMS, DMD, James Webb Space Telescope

\section{INTRODUCTION}

The James Webb Space Telescope (JWST) is the next generation of space-borne astronomy observatory taking over from the expiring Hubble Space Telescope. It is named in honor of NASA's second Administrator and is a joint NASAEuropean Space Agency (ESA) mission. The JWST will carry four scientific instruments that will span the electromagnetic spectrum from $0.6 \mu \mathrm{m}$ to $27 \mu \mathrm{m}$. One of the instruments is the Near Infrared Spectrometer (NIRSpec). The NIRSpec instrument is designed to simultaneously generate and image the spectra from a multitude of carefully selected celestial objects. There are four primary science objectives [1] of the JWST mission: 1) to identify and study the very first bright objects that formed after the Big Bang, 2) to determine how galaxies were formed and how they have evolved from their birth to the present, 3) to study the birth and subsequent formation of stars and planets and, 4) to study the properties of solar systems and locate where conditions for life may exist. The JWST is scheduled to be launched into an L2 Lagrangian orbit ( 1.5 million miles from earth) in 2013. The telescope will have a collecting primary mirror that is 6.5 meters in diameter (over 20 feet) and the NIRSpec instrument will operate at $35 \mathrm{~K}\left(-238^{\circ} \mathrm{C}\right)$. An artist's concept is shown below in figure 1. The Near IR Spectrometer is designed to select up to 100 separate celestial objects at any given time and simultaneously form high (from $1-5 \mu \mathrm{m}$ ) or low resolution $(0.6-5.0 \mu \mathrm{m})$ spectra of each of those objects. The spectra are imaged onto two $2,048 \times 2,048 \mathrm{HgCdTe}$ detector arrays. The requirement to simultaneously observe spectra of multiple objects is an enormous engineering task as compared to observing individual objects one at a time. The challenge arises from the requirement that signal from one object must be kept distinct and uncorrupted by signals from any other objects. To achieve this, a randomly addressable, reconfigurable selection "mask" needs to be inserted between the objects and the detector array. The requirements for this mask are relatively simple, the implementation is very complex. The requirements are:

-Light from selected objects must be transmitted and light from unwanted sources must be blocked

-The mask selection must be reconfigurable and known 
- Over the 5.5 year mission, up to 39,500 MSA reconfigurations may be necessary

- The device must withstand the rigors of an Ariane 5 rocket launch

- The device must be extremely reliable
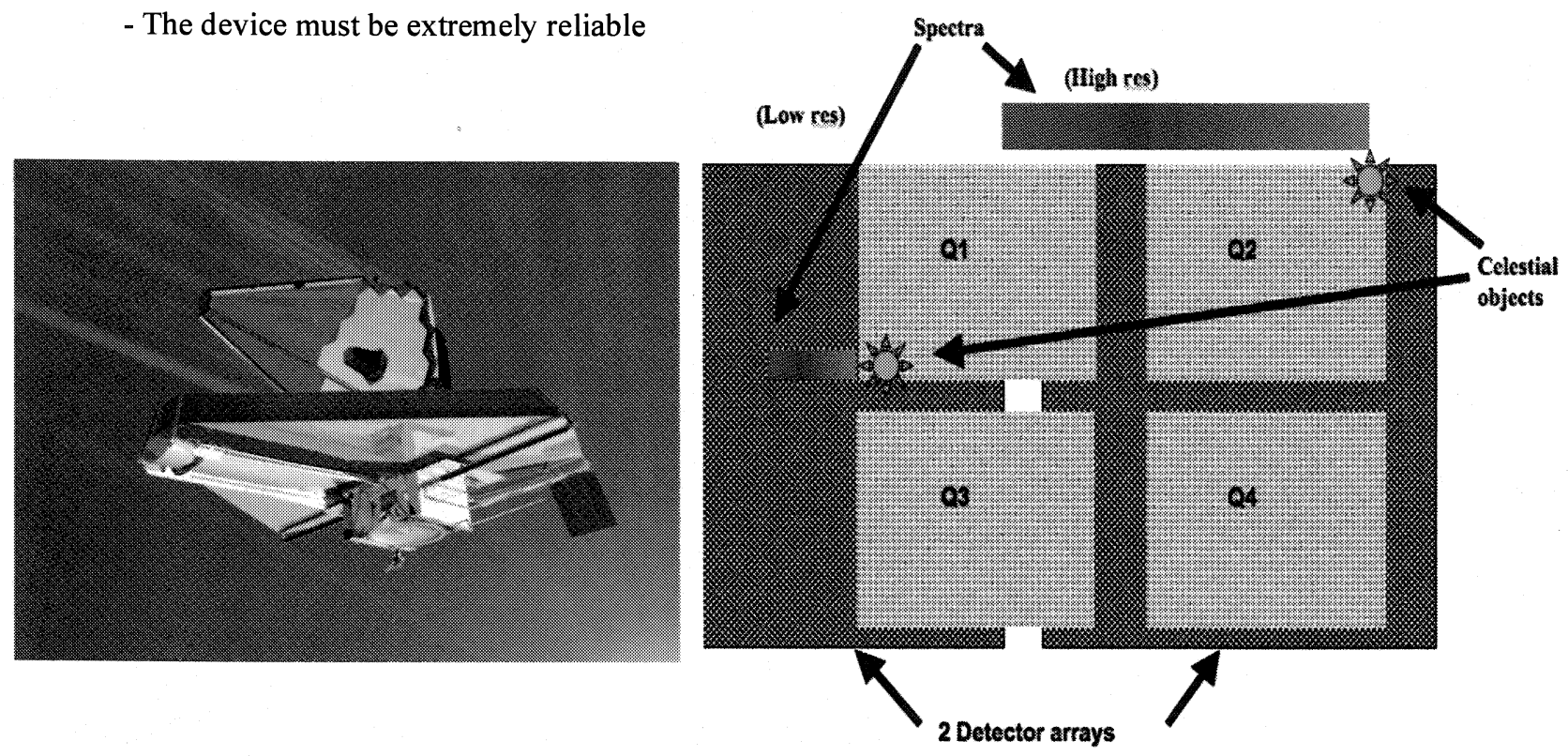

Figure 1. An artist's concept of the James Webb Space Telescope (left). In the diagram at the right four microshutter arrays allow light to pass through from selected objects. The light is deconstructed into a spectrum for each object along one row. Shown above is a sample high and low resolution (res) spectrum.

The first requirement is extremely important in ensuring that the science data can be collected. Light being transmitted through one cell is subsequently formed into a spectrum. This spectrum is dispersed along the length of the row, one cell width wide. The diagram in figure 1 illustrates this dispersion for high and low spectral resolution. A very severe constraint that is derived from this method is that there can be no light from any other sources transmitted through any cells in the row where an object is selected. If there is any light leakage through a cell in the same row as the object of interest this signal will be superimposed on the spectrum essentially destroying the science information of the intended object. The NIRSpec science team has placed a lower limit of 2,000 as the ratio (defined here as the contrast ratio) of transmitted signal to blocked signal with a goal of $>10,000$. A further requirement was the necessity to image 2,500 galaxies over the course of the mission and NIRSpec would be allotted $1 / 3$ of the 5.5 year mission to accomplish this goal. Conventional methods of spectrometry would be very cumbersome (mass, power and limited multi-object simultaneous imaging). Two technologies were considered. The first was the digital micromirror technology that had been developed by Texas Instruments [2]. However, the high contrast ratio requirement and the cryogenic operating temperature were determined to be highly problematic for the micromirrors although the technology was relatively mature for commercial applications. The concern was that since the micromirrors would be steering the optical signal by reflection that it would be very difficult, if not impossible, to ensure that unwanted signal from bright objects, also reflected but at a slightly different angle, would not be scattered into the desired object signal path. This concern was further complicated by the need to operate at a temperature of $35 \mathrm{~K}$ where distortion could further aggravate this problem. A second technology, still in its conceptual phase, was also considered. The microshutter concept was considered and ultimately selected because, unlike micromirrors, the desired optical signal would be transmitted and the unwanted optical signals reflected. The reflection of this unwanted signal ensured that it would not enter into the desired transmitted optical beam path [3]. The challenge for microshutters was quite daunting. Since this technology had not been previously developed all the research and development had to occur and then immediately transition into space flight qualified hardware. The challenge was made even more difficult once the implications from science requirements were translated into device specifications. This paper will describe the system requirements, the key subsystems, testing methods and subsystem performance. 


\section{SYSTEM OVERVIEW}

The key system level requirements that a microshutter-based device needs to meet are listed below:

- The device must operate at a temperature of $35 \mathrm{~K}$

- Up to 100 objects may be simultaneously imaged, mission requirement of 2,500 galaxies

- Spectral wavelength coverage; 0.6-5.0 $\mu \mathrm{m}$

- $9 \operatorname{arcmin}^{2}$ field of view (FOV)

- Total number of reconfigurations: 39,500

- Radiation hard to 100,000 rads

- Mission lifetime: 5.5 years

From these mission requirements a set of overarching parameters can be determined for the microshutter array:

- Shutter dimensions: $\sim 100 \mu \mathrm{m} \times 200 \mu \mathrm{m}$

- Array format: $171 \times 365$ for a total of 62,415 shutters

- 4 arrays necessary to cover the FOV

- Contrast $>2,000$; goal $>10,000$

Probably the most important requirement was the specification of the number of shutters that could be either failed in a closed position, or worse, failed in an open position. The original specification allowed up to 5 rows (171 side) to have one or more failed open shutters at the beginning of the mission and up to 10 rows at the end of the mission. A shutter is considered failed open if the contrast specification of 2,000 is not met. Therefore, a shutter does not have to be missing to be considered a failed open - it can be in a partially stuck open condition or have a small processing defect resulting a clear spot, for example. Once a row had a failed open shutter that row was considered useless so additional failed opens in that row were essentially inconsequential. It would only take 5 shutters out of the 62,415 to fail open to render the entire array non-compliant. The shutter yield in a given array had to exceed $99.99 \%$. The reason for this tight

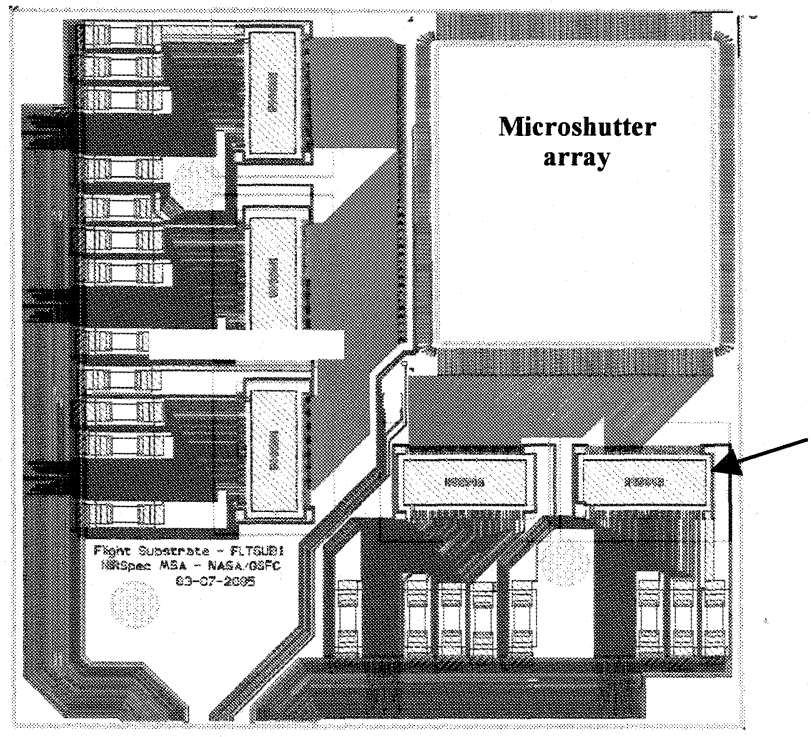

Figure 2. Schematic layout of the substrate. The substrate provides the electrical and mechanical interface between the microshutter array and the NIRSpec specification is that once a row has an open shutter light transmitted through that cell will be superimposed on the spectra generated from an imaged object in the same row. The specification for the number of failed closed shutters is far more lenient. At the beginning of the mission $5 \%$ of the shutters could be failed in the closed position $(\sim 3,100$ shutters) and at the end of the mission $10 \%$ of the shutters could be failed closed. The reason for the large difference between the failed open and failed closed specification arises from the mission. For

1 of 5 high voltage CMOS addressing chips any given astronomical observation at most, only about 100 shutters would be opening in two adjacent arrays-the other $124,000+$ shutters would be closed. As the development of the microshutter fabrication evolved it became apparent that the failed opens specification was very difficult and subsequently this specification was slightly relaxed [4]. Implicit in the design is that each and every shutter must be independently addressable. In order to cover the field of view requirement, four separate arrays would be required totaling 249,660 individual shutters. These four arrays would then need to be mounted on separate carriers and independently assembled. As the program developed, it became clear that this carrier would be required to perform a major portion of the electrical addressing functions as well as providing the mechanical and electrical interfaces to the microshutter array. The carrier (referred to as the "substrate") design is shown above in figure 2. After a quadrant is assembled with all the components (CMOS die, capacitors, temperature sensors and the microshutter array) it is attached to a titanium mount and epoxied to 3 flexure veins. Once mounted onto this interface plate, the quadrants are free to move in the z-direction but laterally constrained to accommodate any motion that will occur as a result of the 
thermal environment. Figure 3 shows the flexure mounting method and the positioning of the 4 quadrants onto the base plate. There are two substrate orientations - a left-handed and right-handed version. However, as will be described, the microshutter arrays must also be oriented relative to the substrate. Consequently, the substrate quadrants are specific for a given location, they cannot be interchanged.
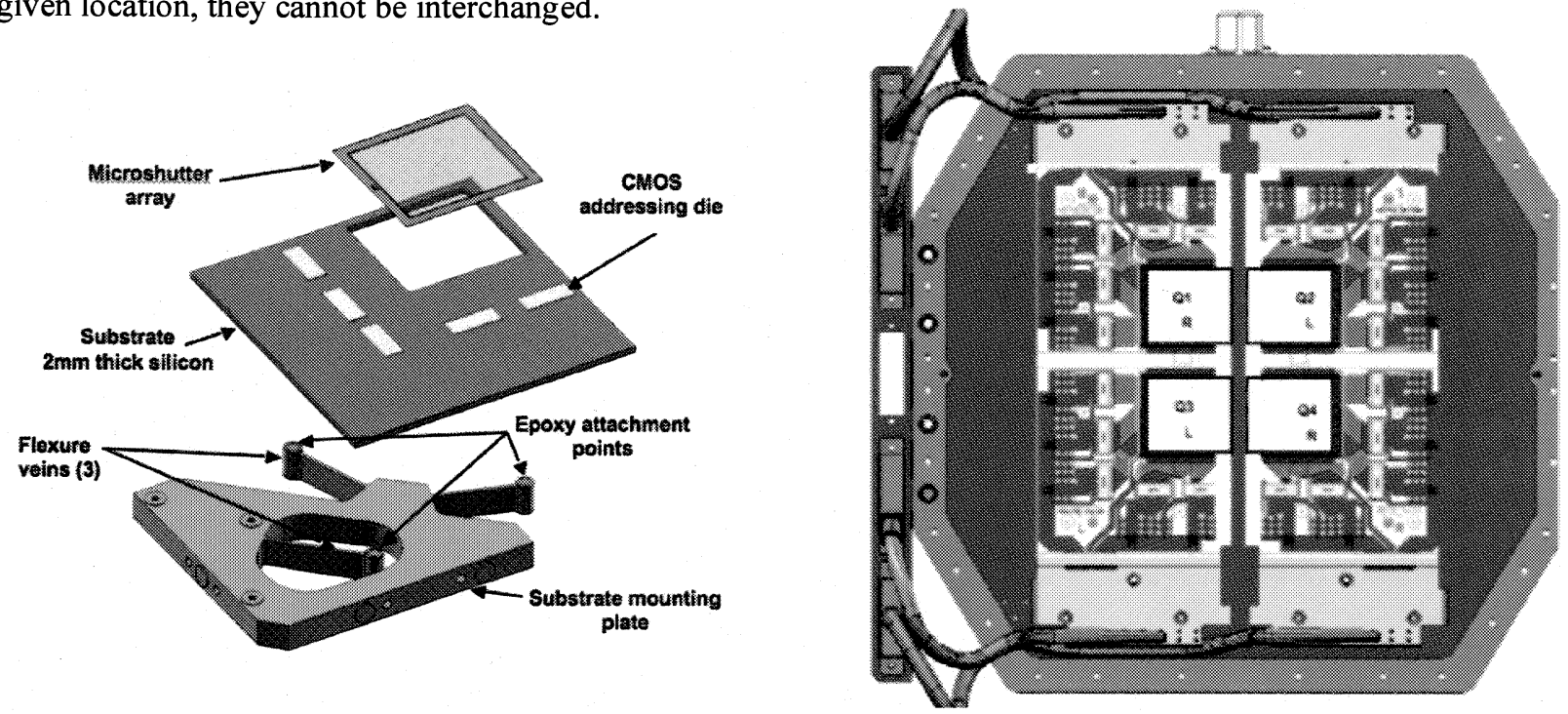

Figure 3. The assembly of the microshutter array and substrate to the flexure plate is shown in the left view. The right view shows the layout of all 4 quadrants on the NIRSpec base plate. The base plate temperature is $35 \mathrm{~K}$.

All the subassemblies, which includes the CMOS addressing chips, microshutter array, silicon substrates, connector board, assembled quadrant and the mosaic of quadrants are rigorously and continuously tested throughout its manufacturing and assembly. Each of the components, including the custom CMOS chips is extremely valuable by the time they are ready to be assembled. A defect in any one of the components that goes undetected is a disaster especially if it is only discovered after a microshutter array has been hybridized onto the substrate. A thorough and comprehensive device screening and monitoring program was, and continues to be, absolutely essential. Additionally, post-microshutter fabrication risk mitigation and performance restoration techniques have been implemented.

\section{MICROSHUTTER ARRAY SUBASSEMBLY}

The development and fabrication of the microshutter arrays has been reported by Li [4]. The precise details of the processing steps are subject to ITAR control. There are many components to the microshutter array. However, each microshutter cell contains a ferromagnetic coating on the shutter, an electrode on the shutter and an electrode on the
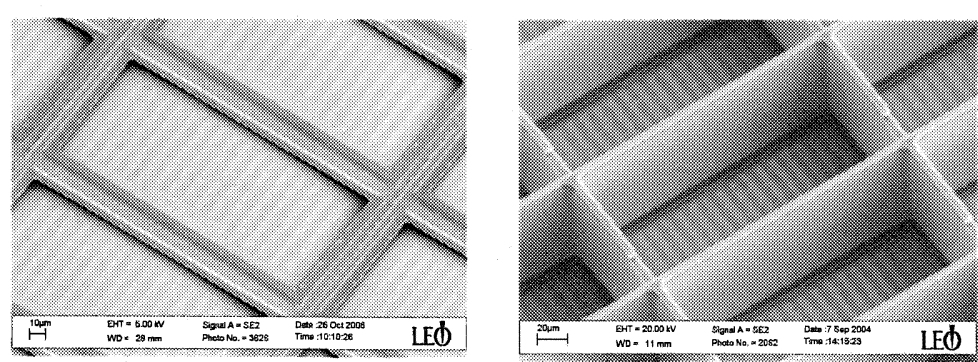

Figure 4. SEM images of the front (left) and back (right) of a few microshutter cells. back wall, a torsion bar and hinge and light shields. The light shields are an additional feature necessary to block out light that would be transmitted around the edges of the shutter. Scanning electron microscope images of a section of the array are shown in figure 4. The microshutter operating method is relatively simple in theory. A positive voltage is applied to the back wall of the shutter cell via electrodes that run along the bottom of the back wall. At the same time a negative voltage is applied to an electrode on the shutter front surface. An external magnet is brought into 
close proximity to the shutter rows and the magnetic field rotates these shutters from their horizontally closed state to their vertical or open position. The potential difference between the surface of the shutter and the back wall, which the shutters are now in close proximity to, pins (or latches) the shutter to the back wall once the magnetic field is removed. This process is illustrated in figure 5.

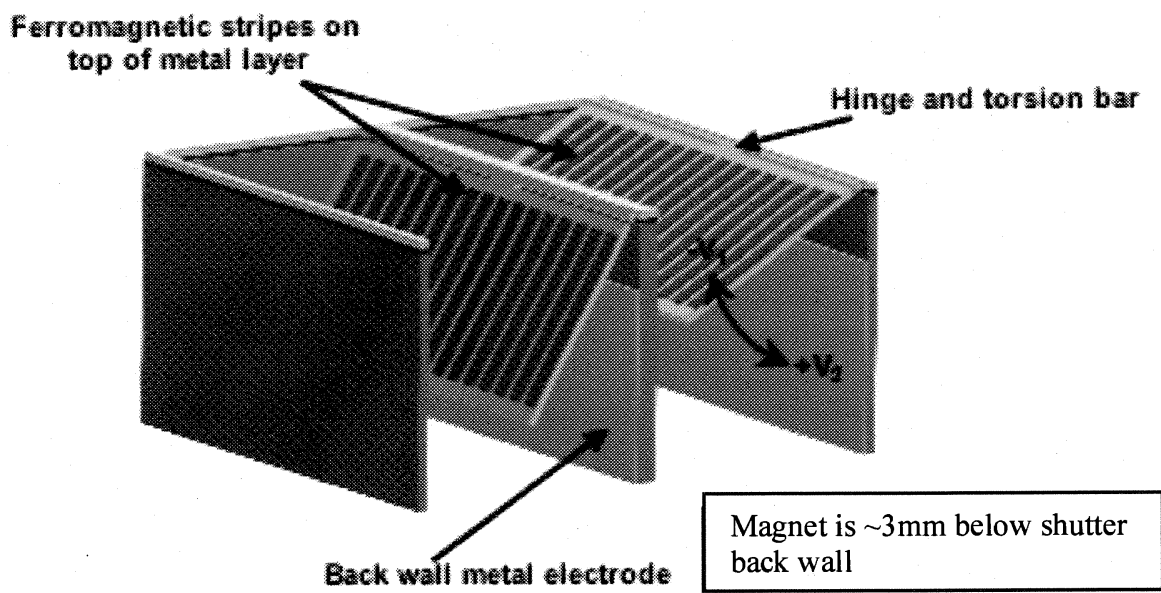

Figure 5. Schematic illustrating the microshutter actuation and latching. In this image the magnet would be approximately $3 \mathrm{~mm}$ below the back wall frame.

The overall physical specifications for the microshutter array are:

$\begin{array}{ll}\text { - Format: } & 171 \times 365 \\ \text { - Pixel Size: } & 105 \times 204 \mu \mathrm{m} \\ \text { - Shutter Dimension: } & 87 \times 186 \mu \mathrm{m} \times 0.5 \mu \mathrm{m} \\ \text { - Opening Area: } & 76 \times 175 \mu \mathrm{m} \\ \text { - Wall Thickness: } & \sim 10 \mu \mathrm{m} \\ \text { - Wall Depth } & \sim 120 \mu \mathrm{m} \\ \text { - Torsion Bar Dimension: } & \sim 190 \times 2 \times 0.5 \mu \mathrm{m} \\ \text { - Open Fill Factor: } & 62.1 \% \\ \text { - Outside Frame Width: } & 3.1 \mathrm{~mm} \\ \text { - Operating Temperature: } & \sim 35 \mathrm{~K} \\ \text { - Number of Rows Allowed with a Failed Open Shutter: } & \sim 5 \\ \text { - Indium bump processing compatible } & \end{array}$

The shutter is extremely thin and very susceptible to changes in shape as a function of temperature. It is necessary that the shutters not be overly bowed when they are being electrically latched. Too much bowing causes the edges of the shutters to dig into the wall or hit the light shields and after many thousands of cycles this would lead to failure. Much effort was expended to keep the shutters flat over the entire $300 \mathrm{~K}-35 \mathrm{~K}$ temperature range. However, it was finally agreed to make the shutters flat at the operating temperature of $35 \mathrm{~K}$ and severely curtail any room temperature operation. This was a hard but necessary trade-off. Finally, the array had to be fabricated to be compatible with indium bump bonding. This was the method that was selected to attach the microshutter array to the silicon substrate. Previously, gold stud bump bonding was utilized but concerns over the long-term stability particularly through multiple cryogenic cycles were raised and this approach was supplanted by an integrated indium bump bonding process. Fabricating a microshutter array requires almost three months of processing, some of which is very tedious such as front-to-back mask aligning. The fabrication is performed in the Goddard Detector Development laboratory that is a well-equipped facility for fabricating small volumes of unique devices. As a consequence only eight 4 " diameter wafers (each containing one array) are processed in a run. The manpower and facility investment quickly adds up and techniques have been developed and implemented to enhance the probability of a space flight grade array emerging from fabrication. Wafer probing is performed during various phases of the array fabrication to ascertain if there are shorts between metal traces (which can be repaired in-situ) on both the front and back of the wafer. Bowing measurements are made to evaluate the shutter flatness from room temperature to $\sim 30 \mathrm{~K}$. The arrays are subjected to an external uniform magnetic field ranging from $0 \mathrm{~T}$ to $0.6 \mathrm{~T}$. This test ensures that 
the shutters are free to move. If there is residue from the final array processing, additional cleaning can be performed and the array can then be retested in the external magnetic field to confirm freedom of motion.

\section{SUBSTRATE SUBASSEMBLY}

When first conceived the substrate was merely intended to be a simple vehicle for mechanically supporting the microshutter array and providing an electrical interface between the microshutter array and the external control electronics. It rapidly became evident that this structure was going to be far more complex and difficult to fabricate than our initial estimates indicated. There were some basic requirements that provided boundary conditions on the construction of this interface plate. It had to be thermally compatible with the microshutter array, it had to be thermally conductive, it had to be compatible with integrated circuit (IC) processing so that high density metal traces could be patterned and it had to be micro-machineable so that an optical window could be created. These requirements are met by using a silicon substrate that is $2 \mathrm{~mm}$ thick. Once we selected silicon of this thickness virtually all IC foundries responded that this thickness of silicon was incompatible with their standard processing parameters. One vendor attempted to fabrication but was completely unsuccessful. Consequently, the development of this substrate became a highly customized Goddard in-house effort. The technical requirements for the substrate that ultimately evolved are listed below:

Single crystal silicon, 6-inch diameter wafer

Thickness of the silicon wafer $; 2.0 \mathrm{~mm}$

Active area of the substrate; $3.6 "$ x 3.6 "

Two integrated heaters capable of handling 3 watts each

Opaque to IR in vicinity of the microshutter array

Two levels of metal traces

Two levels of contacts including an isolated substrate contact

Indium bump bonding compatibility

Hybridized CMOS (custom) ICs

Precise formation of a window

These requirements, although they may seem relatively innocuous, translate into some very stringent manufacturing parameters. Since the active area of the substrate would consume a 3.6-inch square on the 6-inch diameter silicon wafer and that over 2,300 traces (signals, interleaved grounds and miscellaneous) defects from in the materials and arising from the processing had to be kept to almost zero across the entire area. The details of the wafer processing are covered in the paper by Franz et al in this session. As in the fabrication of the microshutters experience led to numerous risk mitigation techniques. The main problem was shorts between the two metal layers arising from a random fault in the silicon dioxide insulating layer. Since the surface area of this very large die is so vast, even a few oxide faults were problematic. In fact, the one foundry that initially attempted to fabricate this structure was overwhelmed with interlayer shorts. The general fabrication sequence is:

- Oxidize wafer

- Deposit/pattern polysilicon

- Oxidize polysilicon

- Pattern 1st level of contacts

- Deposit/pattern metal

- Open 2nd level of contacts

- First probe test/metal trace repairs

- Second probe test/metal trace repairs

- Final probe

- Indium base layer deposition

- Indium bump deposition/pattern

- Aperture patterning and window formation

- Dice substrate from wafer

- Aluminum deposition on inside edge of the window opening 


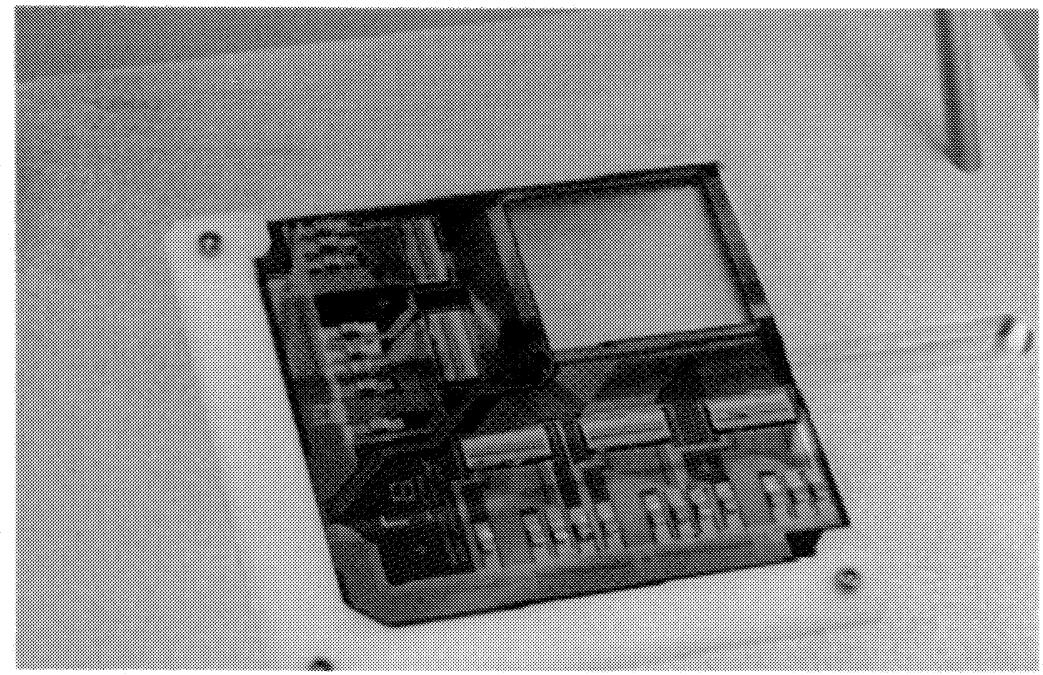

Figure 6. Photograph of a substrate with the HV584s, temperature sensors and capacitors mounted.
Five high voltage addressing/driver chips are mounted on the substrate that select and apply voltage to the microshutter array rows and columns. These chips are rated to \pm 40 volts. The arrays typically require latching voltages in the 25-34 voltage range. The insulating oxide layers on the substrate must withstand high voltages on the metal traces. Often, an oxide fault does not appear at low voltages but does manifest itself at elevated voltages. Therefore, any probing that is performed needs to be able to deliver voltages well in excess of 40 volts. During the fabrication of the substrate the wafer is probed and if there are any shorts or opens they can be photolithographically repaired before they become irretrievably buried. Since these oxide faults are random they can affect virtually any combination of the metal traces. As the substrate gets closer to completion its intrinsic value rises dramatically so the screening procedures become more comprehensive. The first probe test occurs after the aluminum deposition but prior to indium deposition. During this test 100 volts is applied to adjacent input pads and then to all combinations of these pads (two at a time). Each pad is also probed to ensure isolation from the substrate. A second low vow voltage test is performed to verify the continuity of the metal traces from the pads to the trace destination. During this test a repeat of the combinatorial shorts test is again performed. If any traces need to be repaired they are done so at this time and then the substrate is again fully tested. Once the substrate is certified it can resume the final processing which includes the deposition of the indium and bump formation followed by the deep reactive ion etching of the window region. The final processing step is to cut the substrate out of the wafer. The substrate is again subjected to the same set of tests plus an additional test to ensure all the output lines $(171+365)$ are contiguous and short free. Once the substrate has been thoroughly tested and found to be acceptable it is populated with the discrete components and then wire bonded. The HV584 die, temperature sensors and capacitors (for AC decoupling) are all attached with epoxy. A populated substrate is shown above in figure 6 . The temperature sensors and capacitors are wire bonded but the wire bonding for the HV584s is performed sequentially and functionally tested after each die is completed. There is a small chance that a previously screened HV584 may have become damaged. Once all the HV584s are wire bonded and if there is a problem with one of the die, the identification of the defective die can be very difficult and time consuming (depending on the defect). This is a lesson we learned all too well in the early phases of this project and the problem became enormously more complicated when there are defects in the substrate as well. To mitigate this concern, a temporary "daughter board" is wire bonded to the edge of the substrate. This daughter board provides the interface between the external electronics and the substrate. One edge of the daughter board is temporarily wire bonded to the substrate and the other edge has a 100-pin connector. After the wire bonds are attached to the first HV584 voltage and current levels are screened. Any anomalous readings warrant closer scrutiny. If the operating voltages and currents are nominal, the next HV584 is wire bonded and the current/voltage characteristics are again monitored. This process is repeated until all the HV584s are wire bonded. A final functional test is

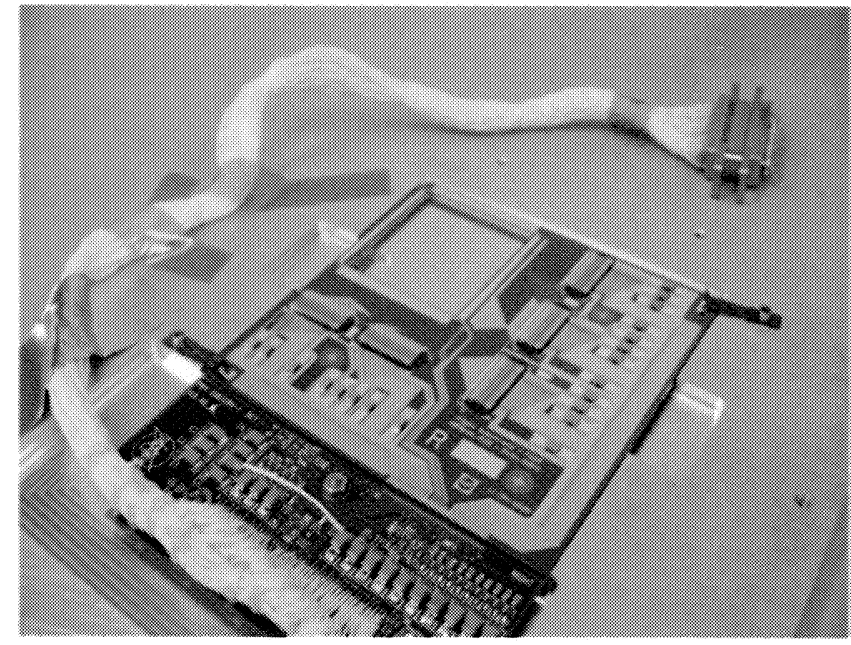

Figure 7. The daughter board with connector is temporarily attached to the substrate. 
performed on the assembled substrate which now includes verifying the digital input addressing (with a readback) and probing each of the 536 high voltage output lines. There is still an opportunity to perform repairs. If for some reason we discover an anomaly in one of the HV584s, it can be deactivated and a new die epoxied on top of the defective one and wire bonded to the substrate. However, with the comprehensive screening tests that are now routine, we have not had to make this kind of repair. If it turns out that one of the output lines from the HV584 to the array is defective a decision can be made to use as is knowing in advance that there will be some shutters that will not be addressed and will remain closed. This is a tradeoff that can be made depending on the destination of the final assembly (Flight, engineering test unit, practice etc.). A photograph of a daughter board attached to the substrate is shown in figure 7 . Once all the component assembly and testing is completed the daughter board is decoupled from the substrate and the substrate is prepared for the indium bump bonding of the microshutter array. The microshutter array has electrodes on both the front side and backside. The array is bump bonded on the shutter side to the substrate and the backside is wire bonded to the substrate. Including the HV584s and the other discrete components on the substrate there are almost 1,500 wire bonds per each assembly.

\subsection{High voltage CMOS addressing/driver chip}

The microshutters are controlled by applying then selectively removing a differential voltage of approximately \pm 30 Volts. Ambient temperature control electronics serially shift data to a set of cryogenic HV584 high voltage drivers mounted on the substrate. The drivers are biased at two separate return potentials to produce the differential voltage. The HV584 microshutter drivers were based on commercially available devices (Supertex HV583) that were redesigned to operate at cryogenic temperatures and withstand radiation environments of up to 200,000 rads. The I-V characteristics were analyzed on process control monitor transistors; data was collected from room temperature to $\sim 30 \mathrm{~K}$ and as a function of radiation dosage. This data was used to modify design rules and the fabrication process and develop a new part. These evolved devices, designed and fabricated by Supertex Inc., are identified as HV584s. A block diagram of the HV584 architecture is shown in figure 8 [6]. The Supertex HV584 is a 128-channel low-voltage (5 V) serial to highvoltage (up to $40 \mathrm{~V}$ ) parallel converter with push-pull CMOS outputs. The part has two data inputs, through which the

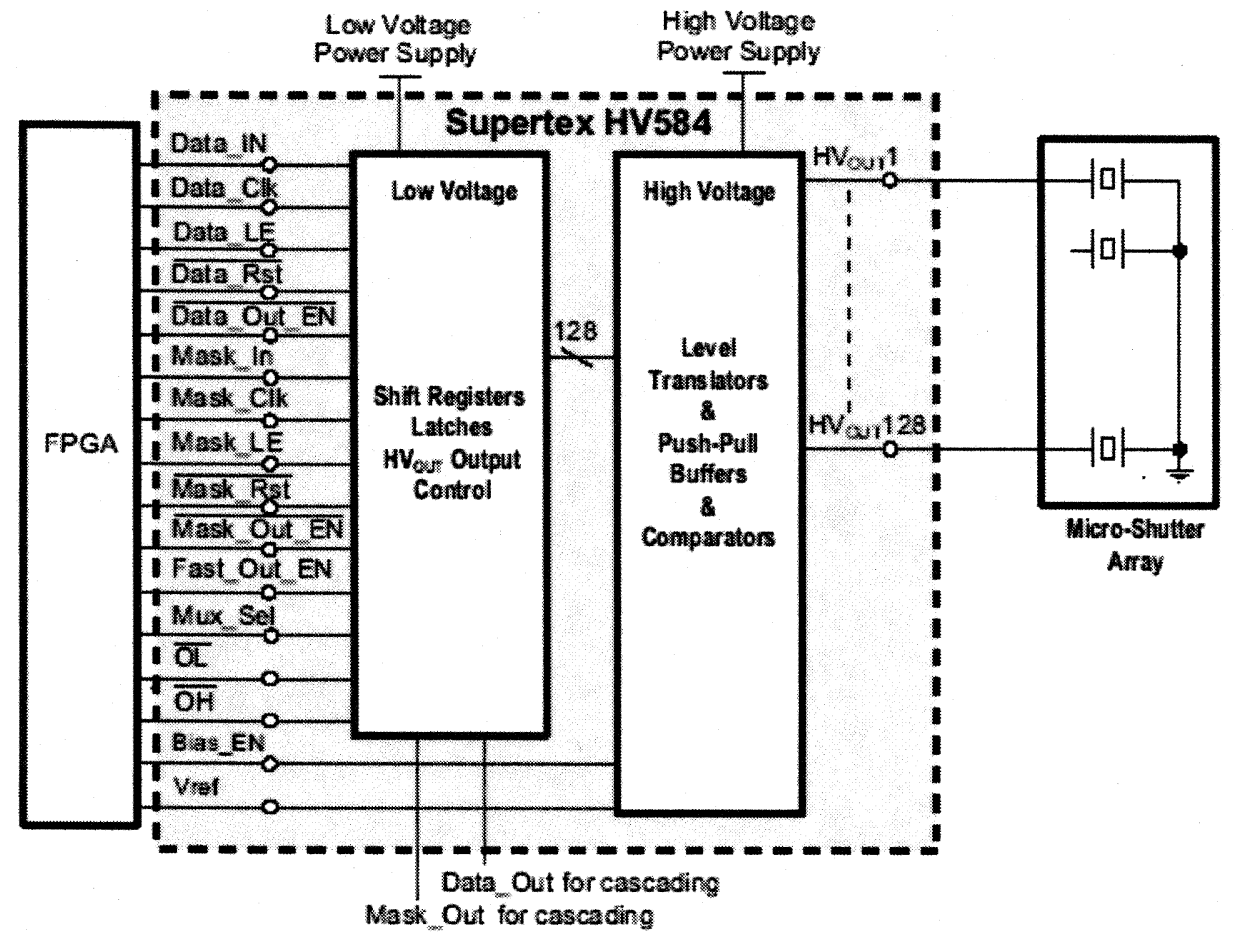

Figure 8. Block diagram of the high voltage CMOS address/driver HV584 IC. data are shifted in during the low to high clock transition. Two sets of 128-bit shift data registers and data latches allow controlling the state of each channel to be either a high, low, or Hi impedance state. The serially shifted data is also available as a digital output. The state of high-voltage outputs can be compared to an analog reference level, latched, and shifted out device allows the input data as well as the high voltage outputs to be verified by serial data feedback. Schmidt trigger input buffers allow stable operation with slow 
rise time input signals due to the thermally isolating (and high electrical resistance) cable. Multiple drivers are concatenated to provide the proper number of channels. Two HV584 devices control the 171 back electrodes and three HV584s control the 365 front electrodes.

\subsection{Mounting of the substrate to mechanical flexure plate}

The fully populated substrate containing the microshutter array is next attached to a titanium plate at three points. The three-point kinematic mounting to flexure arms is necessary to ensure that the differential thermal contraction/expansion of the dissimilar materials do not cause a failure. Multiple design and fabrication iterations were necessary to balance the required bond strength while allowing for adequate motion. The substrate must be precisely aligned to this mounting plate to ensure that the four fully integrated quadrants fall within the overall system optical alignment tolerances. Figure 3 in section 2 illustrates the stack up. The epoxy application and alignment are performed in an Optical Gaging Products (Rochester, NY) optical metrology system. The substrate is aligned to specific datum reference points on the titanium flexure and the epoxy is allowed to set before final cure. The epoxy bond line thickness is precisely controlled. The NIRSpec instrument optical specifications impose very stringent mechanical stability and knowledge requirements on the mounted, aligned quadrant. This becomes even more challenging when the assembly has to occur at room temperature and then be properly aligned once the quadrant reaches its operating temperature of $35 \mathrm{~K}-\mathrm{a} \Delta \mathrm{T}$ of 265 degrees $\mathrm{C}$. The alignment tolerances are $\pm 100 \mu \mathrm{m}$ in each of the $\mathrm{X}, \mathrm{Y}$ and $\mathrm{Z}$ directions. The alignment stability and repeatability of the assembled system are even more stringent since the substrate may be periodically warmed with the integrated substrate heaters. The offset stability requirement in the X-Y plane is $\pm 0.4 \mu \mathrm{m}$ and the offset stability requirement in the $\mathrm{Z}$ direction is $\pm 10 \mu \mathrm{m}$. A cross section of the mounting scheme is shown below in figure 9 along with a photograph of the Optical Gaging alignment system.
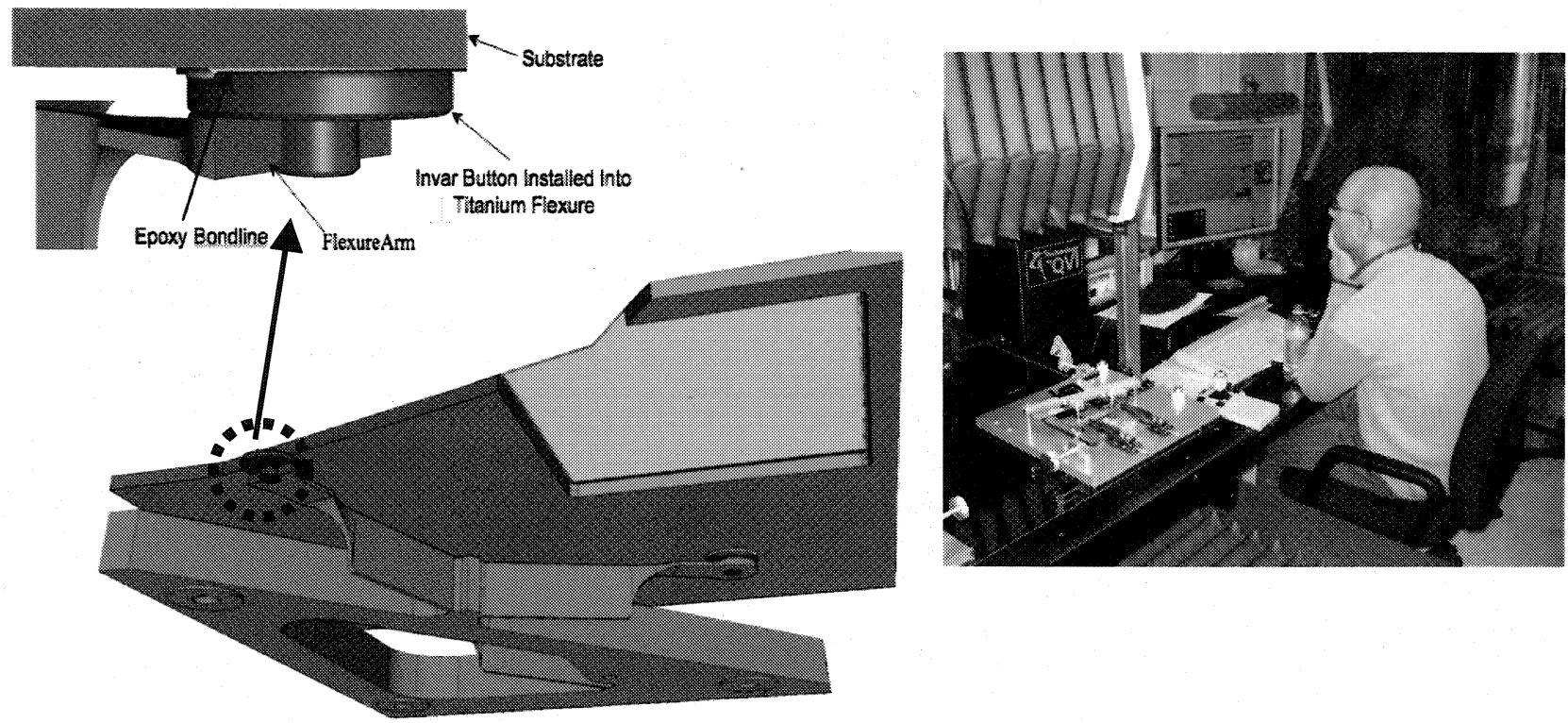

Figure 9. Cross sectional view of the substrate/flexure assembly and exploded view of the bonded flexure joint (top left). The OGP optical metrology system with a substrate in the mounting process is shown in the photograph (right).

Once the substrate is secured to the flexure mount the final assembly and wire bonding of the daughter board to the quad (shown in figure 10 below) and a final electrical test is performed. The fully assembled quad is now ready for cryogenic testing and operation. Prior to installation into the test chamber two more preparatory processes are applied. The first is a thermal-vacuum bake to remove moisture immediately followed by a magnetic actuation in an external magnetic field.

\subsection{Magnetic actuation and addressing of the microshutter array}

Immediately after the bake out the quadrant is placed between the poles of an electromagnet, initially at a slight angle relative to the field direction. A high resolution camera system monitors the entire array as the magnetic field is gradually increased causing the shutters to open. The magnetic field is incrementally increased to $300 \mathrm{mT}$ at which 
point all the shutters should be fully opened. A view of the quadrant between the electromagnet poles is shown in figure 10.
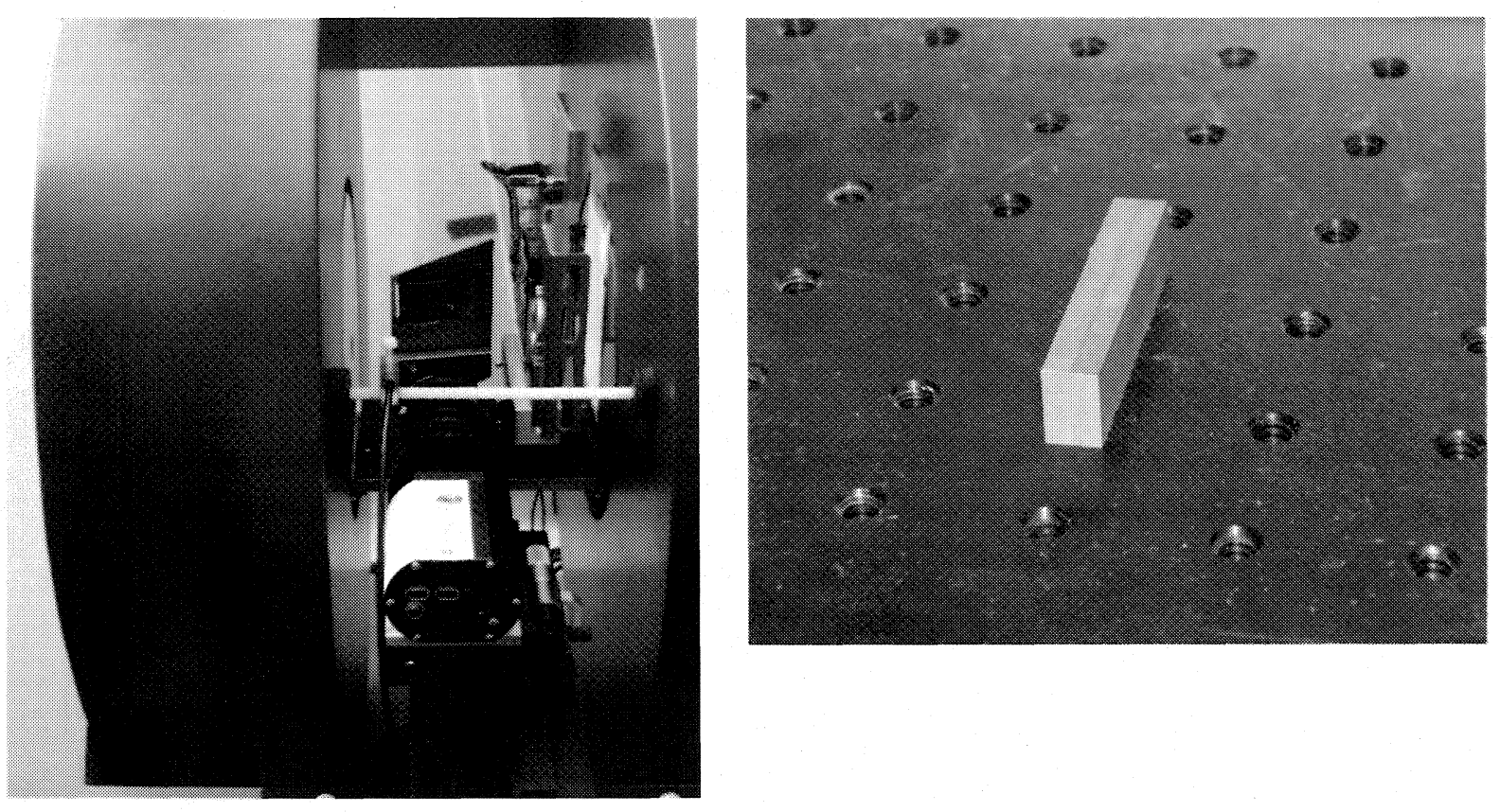

Figure 10. Photograph of the electromagnetic test system with microshutter array quadrant between the poles. The microshutter array is imaged in the mirror and a high resolution camera photographs the microshutters opening as the magnetic field is electrically increased. The photograph on the right is the $8 \mathrm{~mm} \times 10 \mathrm{~mm} \times 45 \mathrm{~mm}$ quadruple praseodymium magnet used in the test system. It consists of two $4 \times 10 \times 45 \mathrm{~mm}$ sections.

This step verifies that the mechanical properties of the microshutter array have not been compromised and that the shutters will open with the same magnetic field as in the test chamber. The magnet used in the cryogenic test system and in the NIRSpec instrument must be able to supply an equivalent magnetic field. Our tests indicated that to achieve a magnetic field strength of $300 \mathrm{mT}$ at a distance of $2 \mathrm{~mm}$ and at a temperature of $35 \mathrm{~K}$ required a unique

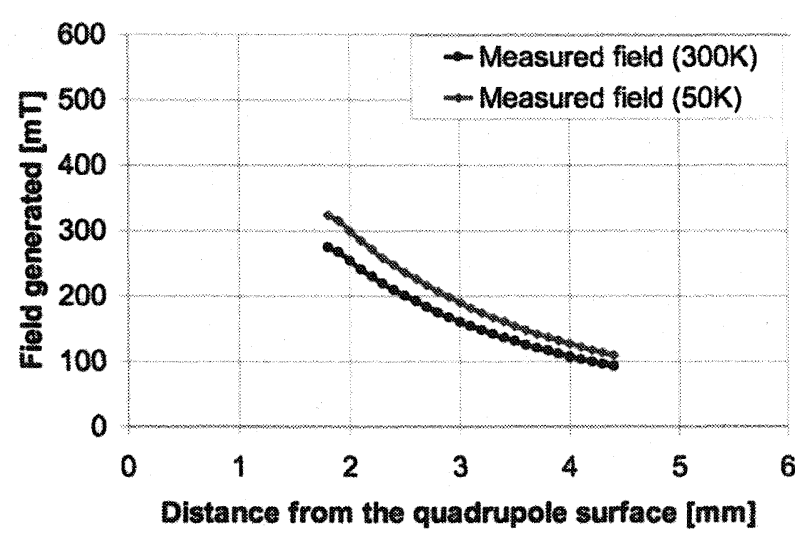

Figure 11. Strength of the magnetic field as function of the distance from the magnet edge. magnetic material configured to enhance the magnetic strength. The magnetic material that was finally selected was based on the rare earth element praseodymium. Other rare earth materials were evaluated but it was determined that at the operating temperature of $35 \mathrm{~K}$ (neodymium for example) would lose magnetic strength whereas praseodymium would actually be slightly stronger $(\sim 20 \%)$ than at room temperature. A plot of the magnetic field strength as a function of distance from the surface of the magnet is shown in figure 11. A field strength of $300 \mathrm{mT}$ is achieved at a distance of $2 \mathrm{~mm}$ at $\mathrm{T}=35 \mathrm{~K}$.

\subsection{Addressing the microshutter array}

The vertical component of the magnetic field opens the microshutters as the magnetic passes by. However, the actual magnetic field geometry determines how the microshutters react in the presence of the magnetic field. When the magnetic is in front of a microshutter the maximum force is exerted by the magnetic field on the microshutter. As the magnetic continues its journey across the array the magnetic field decreases. Once the magnet has passed by a specific shutter the magnetic field decays 
as shown in figure 12. The magnetic field exerts no force on the microshutters once the lateral distance from the center of the magnet $>10 \mathrm{~mm}$ from a given shutter. This is the minimum distance that the magnet must be parked after the array has been actuated so as not to interfere with a configured array. The shape and intensity of the magnetic field also determines the electrical addressing synchronization with respect to the magnetic and its location relative to the column of shutters that is being addressed as the magnet scans over the array. The magnet scan speed ranges from very fast $(4 \mathrm{~Hz})$ to relatively slow $(0.05 \mathrm{~Hz})$. The scan speed in the NIRSpec instrument will be $0.1 \mathrm{~Hz}$ for two arrays. This translates to a magnetic scan rate of 5 seconds to latch the whole array open and then 5 seconds to configure the array with the desired open/close pattern on the magnet return. In this 5 second interval 365

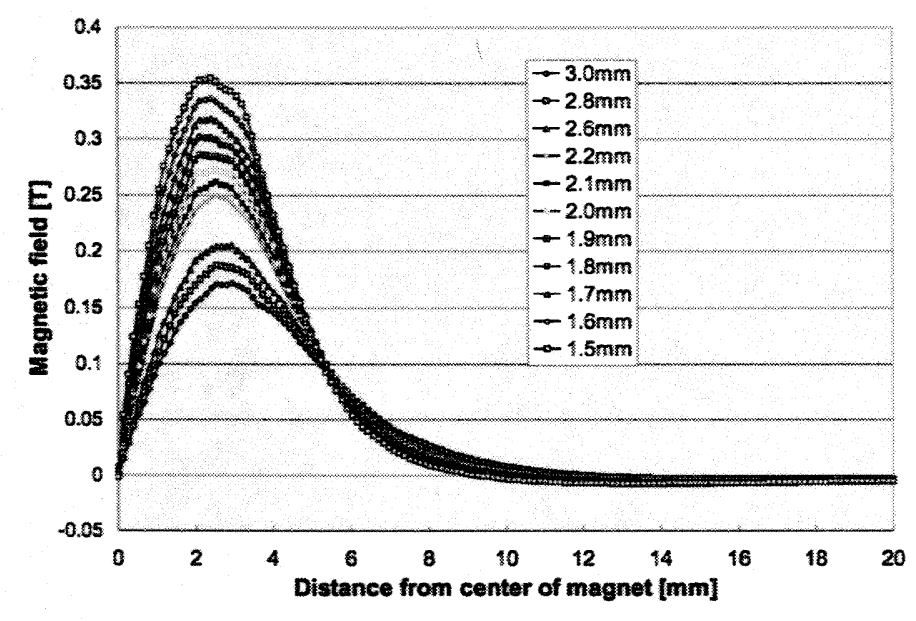

Figure 12. Plot of the magnetic field decay as a function of lateral distance for a given vertical distance. The field is essentially zero $10 \mathrm{~mm}$ from the trailing edge of the magnet.

columns (each column containing 171 shutters) must be precisely addressed. The basic addressing scenario follows three steps;

1.) All the shutters in the array are latched open as the magnetic scans from right to left (arbitrary choice of direction). The shutters are latched with a column voltage of +26 volts and a row voltage of -20 volts. When the magnet has fully traversed the array and is ready to return back to the starting, or home, position both voltages are increased to $+/-32$ volts. The reason for this increase is that during the return addressing cycle the shutters must be held open with only the row voltage since the column voltages will have to go to 0 volts at some point.

2.) the magnet starts to travel back to the home position. Once the trailing edge of into the array, the row voltages are set to

the magnet has passed the first column and is now $2.6 \mathrm{~mm}$ either logic $0(0$ volts $)$ or a logic $1(-32$ volts $)$. The first column voltage is pulsed from +32 volts to 0 volts for 2 ms.
If a 0 is programmed in a given row corresponding to 0 volts the shutter with both the column and the row voltage at 0 volts will spring back to the closed position. If the row is kept at a logic 1 volts it will remain in the open position even though the entire column has been pulsed to 0 volts. After each row of the entire first column is addressed the column voltage is returned to +32 volts. This process is repeated, column by column, as the magnet moves back to the home position until the entire array is addressed.

3.) once the magnet has returned to the home position and the entire array has been configured both the row and column voltages are reduced to $+/-16$ volts. This voltage differential is sufficient to hold the shutters open after they have been latched.

The diagram in figure 13 illustrates this addressing process. The addressing synchronization tolerance, i.e. the position of the centerline of the magnet relative to the location of the column being addressed is shown in figure 14 . This figure illustrates that there is a window of $+/-0.53 \mathrm{~mm}$. This window is due to the side lobes of the magnetic field. One of the important considerations is ensuring that the magnetic field captures shutters as they are released so that they are "gently" returned to the closed position. 


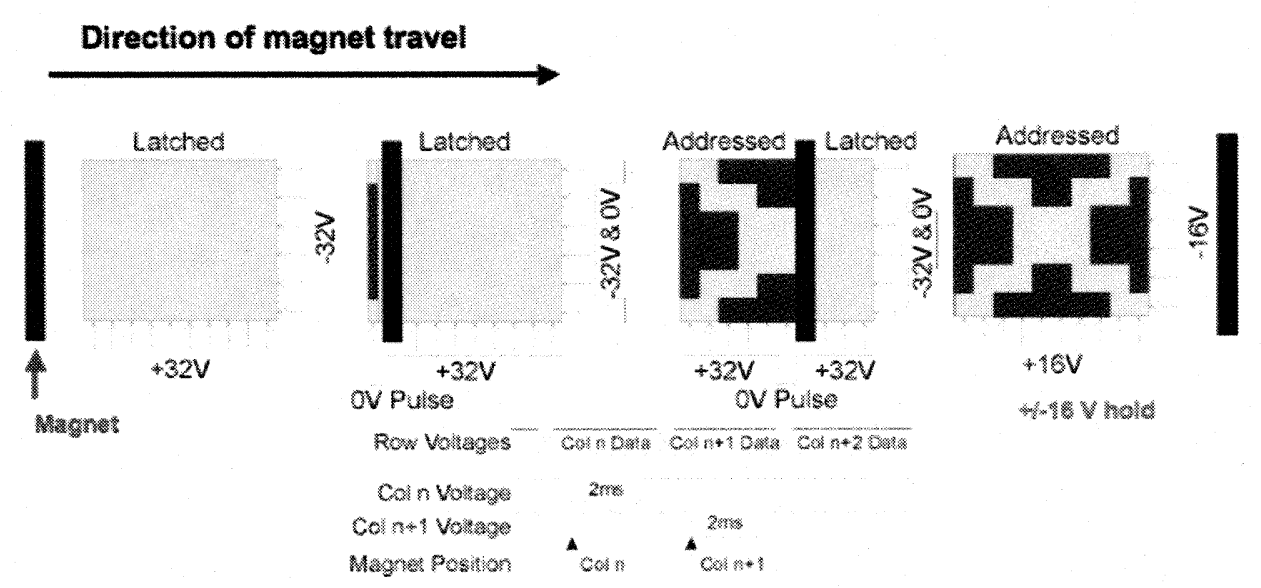

Figure 13. Diagram of the addressing scheme. In this image the magnet has moved from the far right and the entire array has been latched in the open position (in yellow). The magnet is now starting its scan from the far left back to the home position and the array is addressed during this cycle (yellow are open shutters, blue are closed shutters).

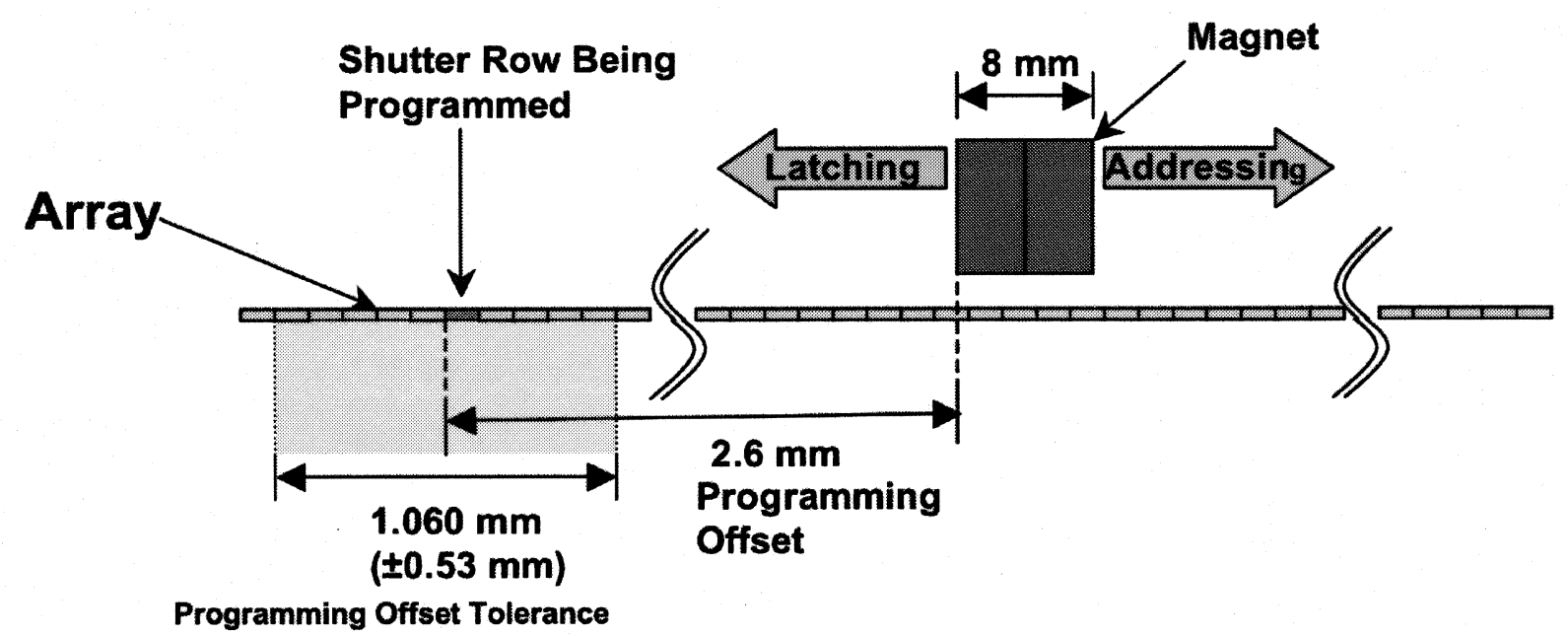

Figure 14. Diagram illustrating the magnetic distance tolerance from a column being addressed. The diagram shows that columns that are within $+/-0.53 \mathrm{~mm}(+/-5$ columns) can be effectively and safely addressed.

There are two possibilities if this gradual release is not ensured. If the magnet is well beyond $2.6 \mathrm{~mm}$ past a given column and shutters in that column are released they will spring back on their own with sufficient force and travel to impinge the over-hanging light shields surrounding the periphery of each shutter frame. Even more severe is that the potential exists for the shutters to experience a magnetic reversal as the magnet passes back over the array that could actually propel the shutters into the shields. This occurs if the shutters are released when the magnet is directly in line with a column. The shutters will survive either of these occurrences for a few cycles. However, after many thousands of cycles severe light shield and shutter damage will result.

\subsection{TESTS AND RESULTS}

After the quadrant has been completely assembled and all the preliminary screening has occurred it is prepared for performance testing in our customized cryogenic vacuum chamber. This chamber contains a magnet on a transport stage, internal and external optics for imaging and contrast measurements and a cold stage that can reach a temperature of $\sim 10 \mathrm{~K}$. Photographs of one of the chambers are shown in figure 15. This chamber is capable of operating two quadrants simultaneously and the magnet can operate at rates of $4 \mathrm{~Hz}$. As part of the qualification program, the array had to be 
opened/closed over 100,000 times so it was necessary to create a system where the magnet could scan the array at high speeds so large number of cycles could be achieved in a reasonable amount of time. Not shown is the computer console that controls the chamber, magnet, electronics, cameras (both visible and infrared), data archiving and analyses programs. Throughout the course of testing one of the better performing arrays over 2,400 images were acquired, over 9,500 files created, over 225 pages of log data and 14 Gbytes of memory were required.
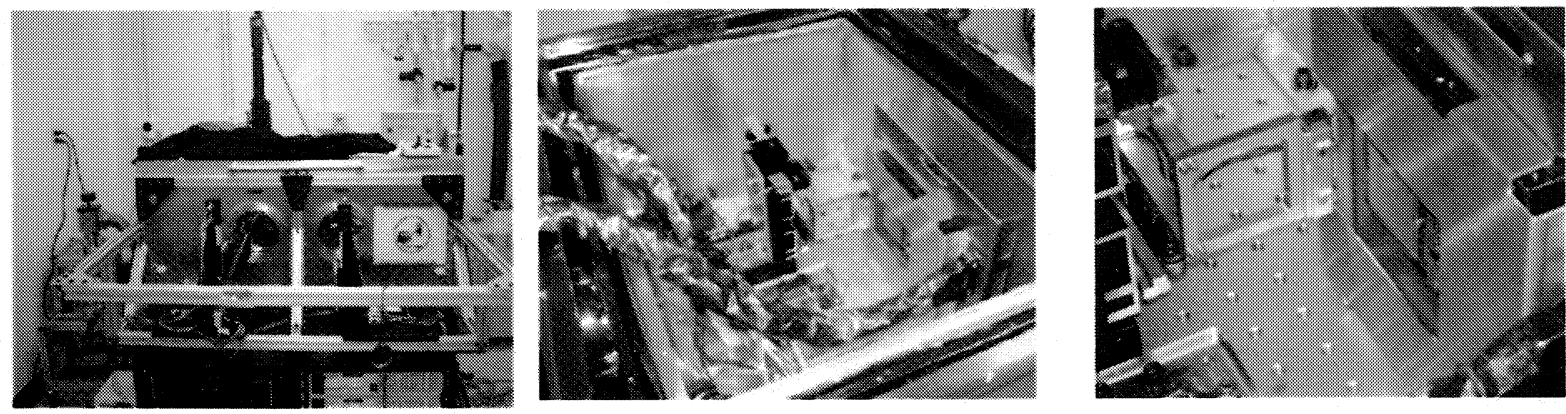

Figure 15. Photographs of the cryogenic test chamber (left), inside of the test chamber (center) and close-up of the microshutter array and magnet transport system (right).

The sequence of testing events occurs as follows:

- Safe-to-mate and HV584 functional test in ambient

- Install quadrant in chamber, activate substrate heaters, establish vacuum and turn on cryocooler

- Once array temperature reaches $\sim 35 \mathrm{~K}$ electrically defective rows/columns are located and masked

- Perform latching, addressing and holding voltage selection/optimization

- Perform test pattern addressing

- Perform $\mathrm{T}=22 \mathrm{~K}$ survival test

- Perform first full array contrast measurement (on every one of the 62,415 shutters)

- Limited life test $\sim 2,000-6,000$ cycles for a flight array; $>100,000$ on test units

- Test substrate heaters to $\mathrm{T}=295 \mathrm{~K}$ (from $35 \mathrm{~K}$ )

- Perform a second limited life test (again, a few thousand cycles)

- Perform a second full array contrast analysis - identify shutters with contrast $<2,000$

- Warm and remove quadrant-permanently seal shutters whose contrast is $<2,000$ (to be discussed)

- Complete retest of quadrant array

Not included in the overall test plan are the tests that were initially performed to verify that the quadrant would survive the severe loads during the launch phase of JWST on the Ariane 5 rocket. The two most severe effects are vibration and acoustics. Suffice it to say that the final mechanical and microshutter design has been qualified to the launch loads plus $3 \mathrm{db}$ of margin [7]. At this time a number of arrays have been tested in a variety of configurations. We will report the results from two such arrays. Recently, we completed testing on an engineering grade quadrant. Once the safe-to-mate, installation and cool down was completed the optimum voltage selection process was performed. First the optimum latching voltage is determined by sequentially increasing the row and column voltages and observing the array performance. The metric is the number of shutters that are latched open with the goal being $100 \%$. However, due to electrical opens and shorts in the array which cannot be quantified until the array is fully operated some rows and/or columns must be deactivated so that even if the shutters are magnetically opened, voltages may be deliberately be in the off state so that these shutters will not remain open once the magnet scans past them. For this particular array a sample of the effect of various voltage combinations is shown in Table 1 . The actual test encompassed voltages ranging from $+/-20 \mathrm{~V}$ to $+/-30 \mathrm{~V}$. From the data in the table it is clear that $+30 /-28 \mathrm{~V}$ was the optimum. However, preference is given to operating at lower voltages if the performance is very similar. In this case the absolute best performance is achieved with $+30 \mathrm{~V} /-28 \mathrm{~V}$. However, by lowering the voltages to $+26 /-20$ only a few more shutters failed to latch. This difference in failed closed is insignificant and the benefit from operating at a lower voltage is preferred. Similar "trial and error" tests were performed to obtain the addressing and hold voltages once the address was set. The selected voltages are summarized in Table 2. From this point a wide 
range of testing options exists. Life tests can be performed to determine how the array will perform over the course

Table 1.

\begin{tabular}{ccccc}
$+\mathrm{V}$ & $-\mathrm{V}$ & \# Open & \% Open & \# Closed \\
\hline 30 & 20 & 59704 & $95.66 \%$ & 2711 \\
30 & 22 & 59709 & $95.66 \%$ & 2706 \\
30 & 24 & 59736 & $95.71 \%$ & 2679 \\
30 & 26 & 59760 & $95.75 \%$ & 2655 \\
30 & 28 & 59860 & $95.91 \%$ & 2555 \\
28 & 20 & 59709 & $95.66 \%$ & 2706 \\
28 & 22 & 59714 & $95.67 \%$ & 2701 \\
28 & 24 & 59722 & $95.69 \%$ & 2693 \\
28 & 26 & 59756 & $95.74 \%$ & 2659 \\
26 & 20 & 59623 & $95.53 \%$ & 2792 \\
26 & 22 & 59638 & $95.55 \%$ & 2777
\end{tabular}

of thousands of cycles, tests to verify precise patterns can be applied, holding shutters open for

days at a time, operating at a range of cryogenic temperatures etc. The failed open specification is determined by the contrast test. Any single shutter that does not have a contrast $>2,000$ (ratio of transmitted light to blocked light) is considered open and the maximum number of failed open shutters is specified to be 14 if there are only 440 failed closed (based on the revised NIRSpec specifications). If there are "0" failed open shutters then the number of failed closed

shutters permitted dramatically increases to $\sim 10,500$. Clearly the emphasis is on the number of failed opens, meeting the failed closed specification is a far simpler matter. The two addressed images below in figure 16 (another array) illustrate this challenge. The images illustrate the difference between failed closed and failed open shutters. The image on the left is a mostly "open" image but the dark lines and spots are shutters that remain closed. There are approximately 2,200 failed closed shutters in this image. The image on the right shows the effect of failed opens in

Table 2.

\begin{tabular}{|c|c|c|c|}
\hline VOLTAGE SUPPLY & LATCH & ADDRESS & $\begin{array}{c}\text { HOLD } \\
\text { (during observation) }\end{array}$ \\
\hline$+\mathrm{V}_{\mathrm{pp}, 365}$ & $+26 \mathrm{~V}$ & $+32 \mathrm{~V}$ & $+16 \mathrm{~V}$ \\
\hline$-\mathrm{V}_{\mathrm{pp}, 171}$ & $-20 \mathrm{~V}$ & $-32 \mathrm{~V}$ & $-16 \mathrm{~V}$ \\
\hline
\end{tabular}

an image that should be completely dark, that is, completely closed. There are $\sim 30$ failed open shutters in this image. The $\sim 30$ failed opens in the image are only counting the obviously failed shutters not all the shutters that failed the contrast specification. Essential to the success of this program is the necessity to eliminate failed open shutters after fabrication is complete. It would be impossible at this time to fabricate any arrays that did not have failed open shutters. Therefore a method has been developed where failed open shutters (based on both the obvious and those that fail contrast) can be "plugged" converting a failed open into a permanently closed shutter. The image of the closed array in figure 16 with the $\sim 30$ failed opens will be used to illustrate this process. The exact $x-y$ location of each shutter to be plugged is recorded (this will be further discussed when the contrast test results are presented). Silicon chips are precisely fabricated in sizes that are discrete increments of a single shutter. A $3 \times 3$ shutter plug would measure $315 \mu \mathrm{m} \times 612 \mu \mathrm{m}$ and would seal or plug 9 shutters with the offender in the center. The images in figure 17 illustrate the success of this process. 

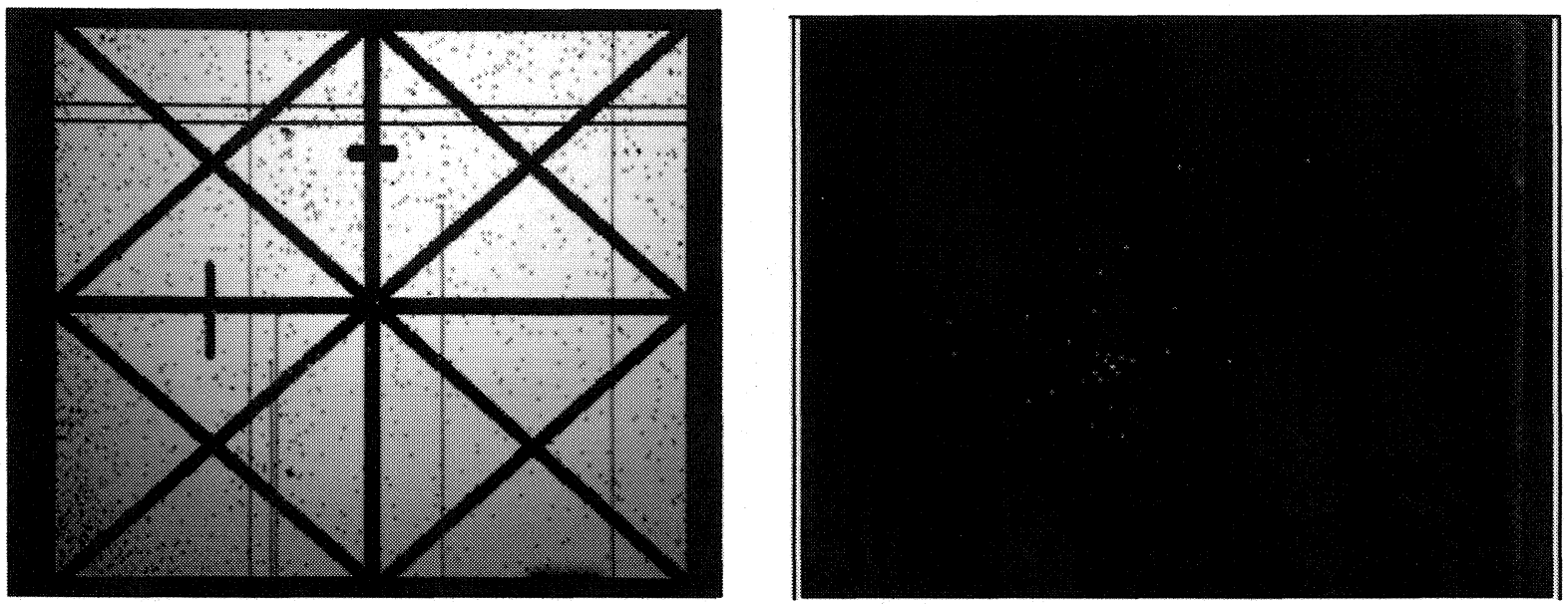

Figure 16. The image on the left is the addressed image of a test pattern that requires most of the 62,415 shutters in the array to be open (white pixels) The black streaks and speckles are lines and individual shutters that remain closed $(2,200$ in this image). The image on the right shows the $\sim 30$ shutters that should be closed but are failed open.

The same array with the $\sim 30$ failed opens shutters received $243 \times 3$ plugs and two $4 \times 6$ plugs. To plug the 30 failed open shutters, 264 shutters were converted to permanently closed shutters. This plugging process took place after this array had already been open/closed $\sim 19,750$ times.
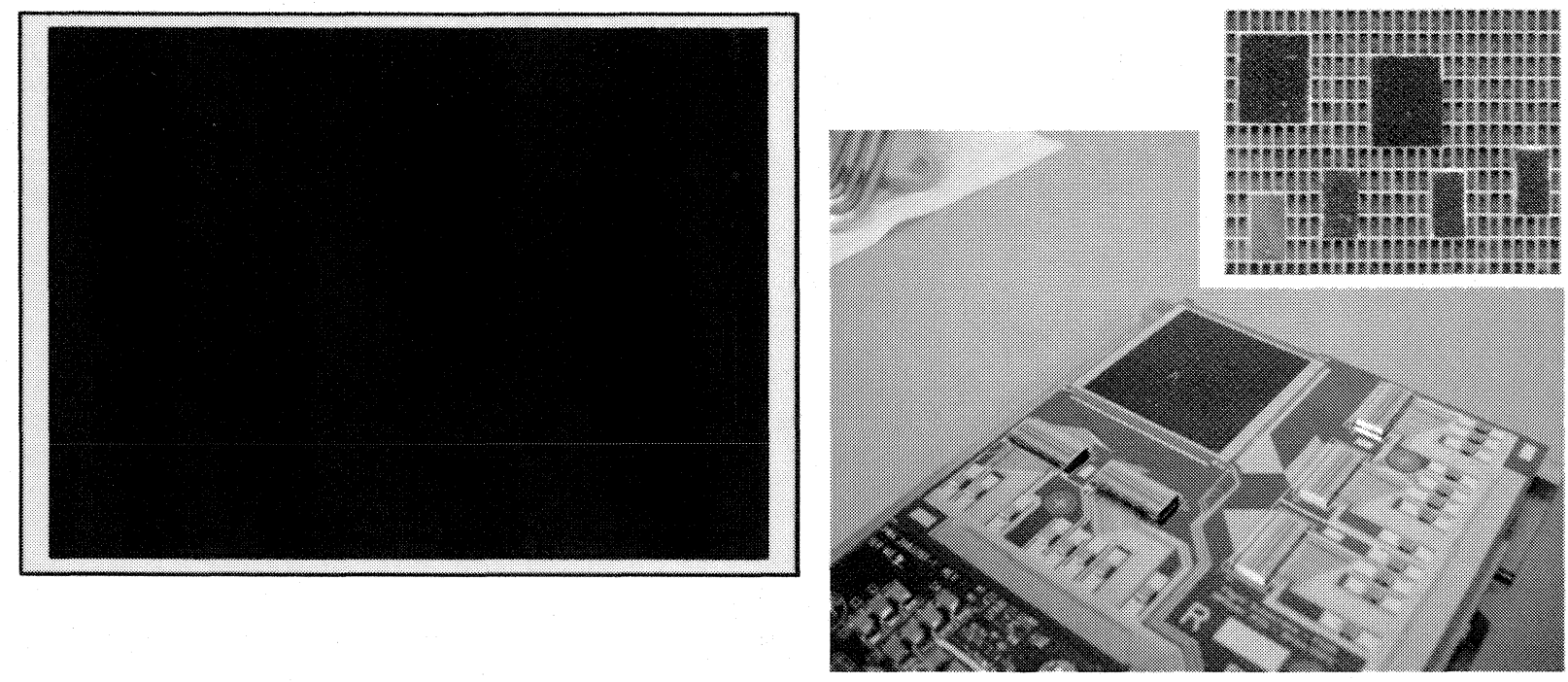

Figure 17. Converting failed open shutters to permanently failed closed shutters. On the right is two photographs illustrating the plugging process. There are 26 plugs installed on the array as shown (they appear as specks on the array). The inset is a higher magnification photograph of six individual plugs. They are precisely manufactured as well as precisely attached to the shutter frames. The image on the left is a repeat of the closed image from figure 16 illustrating the success of the plugging process (failed opens eliminated). 
After plugging the failed open shutters on this particular array it was subjected to a severe 3 -axis vibration, acoustics tests to simulate the launch sequence. The quad was retested in the cryogenic chamber and over 100,000 additional open/close (with some interspersed addressing) cycles were added to this array. About three months were dedicated to evaluating the performance of this quadrant relative to NIRSpec observing scenarios. Some of these tests included holding the entire array open ranging from a few minutes to 24 hours, multiple 15 minute hold-open tests, an actual accelerated mission scenario of $\sim 30,000$ cycles and multiple substrate heating cycles to $270 \mathrm{~K}$. The mission simulation occurred between cycles 52,000 and 82,000. At the start of this test there were now 22 new failed open shutters that had materialized after the plugging process (back at cycle 19,750). At the end of this test there was one additional failed open shutter -an extremely encouraging result. The final test to be described is the contrast measurement. The method for performing this test is essentially to determine how much light passes through an open shutter versus how much light passes through a closed shutter and this ratio is how we define contrast. Tests were performed with both visible $(633 \mathrm{~nm}$ ) and infrared sources (with $\mathrm{H}, \mathrm{J}, \mathrm{K}$ filters). The measurement is extremely sensitive to stray light and the ultimate resolution was determined to be $\sim 100,000$. This means that we could detect a light signal that had been attenuated by a factor of 100,000 . Table 3 below shows a sample of the test results on an engineering grade array. In this particular array there were 81 shutters out of the 62,415 that did not meet the 2,000 requirement. As can be seen from the data there are a few that came close to 2,000 and a few that were very low. The ones that came close might have a pinhole on the shutter, which is letting a small quantity of light pass. The ones that have values exceeding a few hundred are shutters that are stuck in a partially open position. Each failure is identified by location and would necessitate plugging. Since this was an engineering array no plugging was performed. On a high grade, flight quality array there are typically $<50$ shutters that we expect to plug. However, until the complete test is performed this is a variable of considerable concern.

Table 3. A sample of the contrast data on an engineering array

\begin{tabular}{|c|c|c|c|}
\hline Item \# & Row (171) & COL(365) & CONTRAST \\
\hline 1 & 169 & 1 & 1981.82 \\
\hline 2 & 166 & 100 & 171.22 \\
\hline 3 & 165 & 117 & 130.54 \\
\hline & 164 & 1 & 1957.6 \\
\hline 3 & 163 & 1 & 1602.16 \\
\hline & 163 & 52 & 1462.92 \\
\hline 7 & 162 & 127 & 108.36 \\
\hline
\end{tabular}

\subsection{CONCLUSION}

The development of the microshutter-based multi-object selector for the NIRSpec instrument on the James Webb Space Telescope has been and continues to be a very large challenge. The microshutter concept and the ultimate implementation in silicon presented an enormous MEMS fabrication challenge. However, as the arrays became available, from simple test structures to the final flight version, it became readily apparent that other elements of the system would require state-of-the-art engineering and technology most notably the custom high voltage CMOS addressing/driver chips and the wafer-scale silicon substrate on which the microshutter array is mounted. The challenges were further compounded by the very difficult failed open specifications imposed on the microshutter arrays necessary to produce the science data associated with the birth of the universe and its subsequent evolution. At the moment there are 16 flight candidate microshutter arrays waiting to be assembled into quadrants and the hope was that all 16 would be tested quadrants and that there would be an opportunity to select the very best four quadrants for the mission. However, due to schedule and cost constraints we must select four arrays for the flight units and be prepared with 4 back-ups. We must pick the arrays judiciously. 
This final image was made on the array discussed in the previous section at cycle 67,936 during the accelerated life test. It is an image of the Czech Republic Coat of Arms with the NASA logo presented to the President of the Czech Republic.

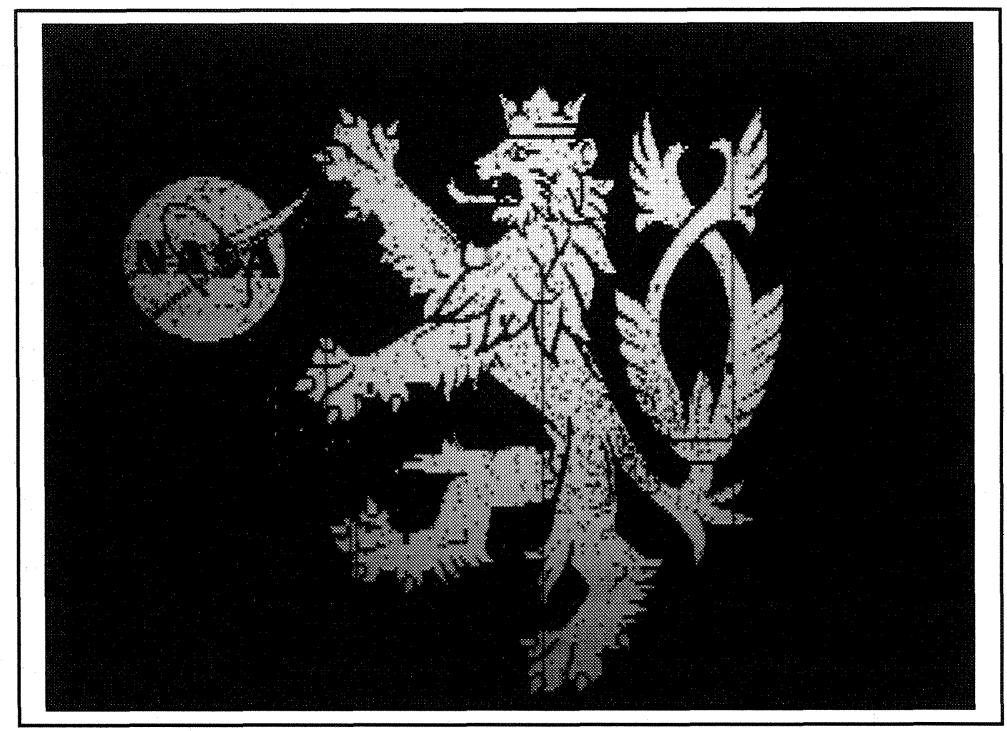

\section{Acknowledgements}

This project has been a very complex, innovative program requiring engineering expertise in a number of disciplines. The list of people working to make this a success is long but the authors would like to thank the following individuals (in no particular order) for their support and dedication to this effort: Steve Snodgrass, Tony Zheng, Chris Zincke, Knute Ray, Nick Costen, Audrey Ewin, John Chambers, Dave Rapchun, Nick Collins, Christine Allen, Mike Beamesderfer, Sachi Babu, Kamili Jackson, Cheryl Albert, Bing Guan, Jay Cho, Joe Santos, Joe Connelly, Leroy Sparr, Dave Sohl, Rob Thete, Charles Tomasevich, Bill Newell, Thomas Hartman, Hal Isenberg, Carol Sappington, John Leitonen, Fred Wang, Theo Hadjimichael, Vilem Mikula, Tomoko Adachi, Eric Lyness, Wayne Ecklund, Rick Arendt, Wayne Landsman, Franklin Miller, Sateesh Bajikar, Ruth Bradley, Kevin Denis, Larry Hess, Ron Hu, Dan Kelly, Todd King, Gunther Kletetschka, Barney Lynch, Timothy Miller, , Brent Mott, Lance Oh, James Pontius, Chris Ray, Knute Ray, Eric Schulte, Peter Shu, Wayne Smith, Rosalind SteptoeJackson, Veronica Valeriano, Liqin Wang.

Additionally, the authors would like to acknowledge the efforts, suggestions and solutions of our European colleagues. Specifically, Peter Jakobsen, Peter Jensen, Giorgio Bagnasco, Jean-Christophe Salvignol and the many other engineers and scientists at the European Space Agency have made important and valuable contributions to this effort and the entire team is grateful for their help and support.

\section{References}

[1] Gardner, J. "The James Webb Space Telescope", Space Science Reviews, vol. 123, No. 4, (2006).

[2] Mado, M. "Fundementals of Microfabrication:The Science of Miniaturization", CRC Press, pp. 308-311, (2002).

[3] Fettig, F., Moseley, S., Kutyrev, A., Orloff, J., Kuhn, J., and Shude, L. "Some Aspects on the Mechanical Analysis of Micro-shutters," in Materials and Device Characterization in Micromachining II, Proceedings of SPIE 3875, (1999).

[4] Jakobsen, P., et al, "The Scientific Impact of Failed Shutters in the NIRSpec Micro Shutter Array" White Paper of the NIRSpec Instrument Science Team Issue 1.0, (2007).

[5] Li, M., et al, " Complex MEMS Device: Microshutter Array System for Space Applications", SPIE Defense and Security (2007).

[6] Supertex Inc., "Product data sheet", (2007)

[7] Q1-11-73 LOG14.doc, " Test log ", NASA/GSFC, pp. 40-41, (2007). 